\title{
Globales Netzwerk der Zumtobel AG wird migriert
}

Equant, einer der führenden Solution Provider für individuelle globale und lokale Kommunikationsanforderungen international operierender Unternehmen, hat mit der österreichischen Zumtobel $A G$ einen Dreijahresvertrag zur Anbindung der Mitarbeiter an das schnelle und sichere Netzwerk von Equant geschlossen.

Der österreichische Konzern Zumtobel AG zählt weltweit zu den größten Anbietern der internationalen Lichtindustrie. Nach der Insolvenz des vorigen Service Providers von Zumtobel, KPNQwest, migrierte Equant den Konzern gemäß des Vertrages innerhalb von vier Wochen in sein IP VPN (Internet Protocol Virtual Private Network).

Die individuelle EQUANT IP VPN-Lösung verbindet 50 Standorte von Zumtobel weltweit. Sie ermöglicht dem Unternehmen, seine IP-, Daten-, Sprach- und Video-Anwendungen über ein einziges Netzwerk zu übertragen. Mit dem MPLS(Multiprotocol Label Switching)-basierenden IP VPN von Equant kann Zumtobel weiterhin seine Produkte den Kun-

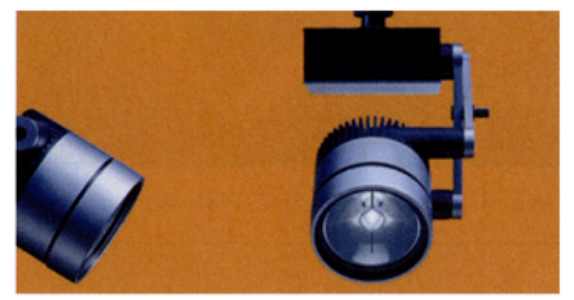

Der österreichische Konzern Zumtobel AG zählt weltweit zu den größten Anbietern der internationalen Lichtindustrie

den und Partnern auf Just-in-time-Basis liefern. Die Any-to-any-Konnektivität des EQUANT IP VPN ermöglicht die flexible Anbindung neuer Standorte. Darüber hinaus bietet sie dem Unternehmen erhebliche Kosten- und Leistungsvorteile.

EQUANT IP VPN bietet eine weltweite, geografisch einzigartige Abdeckung mit uneingeschränkter Any-to-any-Kommunikationsfähigkeit in einem eigenen, privaten und sicheren Netzwerk mit garantierter Service-Qualität. IP VPN von
EQUANT wird mit Übertragungsgeschwindigkeiten bis zu $155 \mathrm{Mbit} / \mathrm{s}$ mit höchster Übertragungsqualität realisiert. Der Service ist durch strenge Service-Level-Agreements für Transit Delay, Packet Loss und Jitter abgesichert. Vier Serviceklassen stehen für die Übertragung von verschiedenen Arten von Applikationen zur Verfügung. EQUANT IP VPN ist multimediafähig und erlaubt sowohl On-netund Off-net-Sprach- sowie Videoübertragung über IP.

Equant bietet multinationalen Unternehmen Netzwerk-, Integrations- und Applikationsdienstleistungen. Sein nahtloses Datennetzwerk verfügt über eine große Reichweite und verbindet wichtige Geschäftszentren in 220 Ländern und Regionen. Das Unternehmen bietet außerdem lokalen Support in 191 Ländern.

Informationen: Equant Austria Telekommunikationsdienste GesmbH, Mag. Gerhard Pix, Heiligenstädter Lände 27c, A-1190 Wien, Telefon +43 (1) 360 37-3310, E-Mail: gerhard.pix@equant.com, Internet: www.equant.com

\section{SpringerInformatik}

\section{Jakob Steuerer, Jørgen Bang-Jensen}

\section{Die Dritte Welle der Mobilkommunikation}

Business-Visionen + Lebens-Realitäten

2002. VII, 115 Seiten.

Broschiert EUR 19,90, sFr 32,-

ISBN 3-211-83804-X

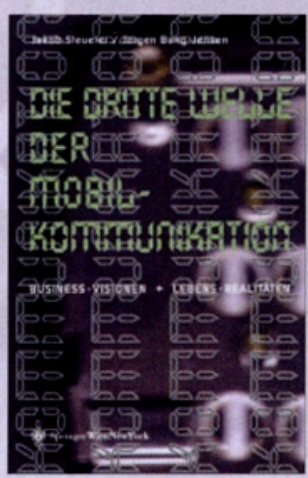

Nach der „Ersten Welle“ Anfang der 90er Jahre, als Handys noch ein elitärer Luxus waren, und der "Zweiten Welle," in der Handys der breiten Bevölkerung zum unverzichtbaren Medium wurden, setzt nun mit einer "Dritten Welle" der finale Gestaltwandel des Handy ein - es empfängt digitale Daten bald genauso selbstverständlich wie Sprache: das Handy dient der Navigation in fremden Umgebungen, sendet und empfängt spontane Bildbotschaften, wird zur digitalen Kreditkarte, verbindet uns mit den Arbeitsabläufen im Büro usw. Die Autoren entführen auch den fachlich nicht versierten Leser auf eine Reise in die nähere Zukunft der Mobilkommunikation.

\section{SpringerWienNewYork}

A-1201 Wien, Sachsenplatz 4-6, P.O.Box 89, Fax +43.1.330 24 26, e-mail: books @ springer.at, Internet: www.springer.at D-69126 Heidelberg, Haberstraße 7, Fax $+49.6221 .345-229$, e-mail: orders @ springer.de

USA, Secaucus, NJ 07096-2485, P.O. Box 2485, Fax +1.201.348-4505, e-mail: orders @ springer-ny.com

Eastern Book Service, Japan, Tokyo 113, 3-13, Hongo 3-chome, Bunkyo-ku, Fax +81.3.38 18 08 64, e-mail: orders @ svt-ebs.co.jp 